\title{
Pulmonary hyalinizing granuloma in HIV/AIDS
}

\author{
Theresa Liu BArtsSc MD ${ }^{1}$, Maggy Kyrollos MD FRCPC ${ }^{2}$, Stephen Kravcik MD MSc (Epi) FRCPC ${ }^{3}$
}

\begin{abstract}
T Liu, M Kyrollos, S Kravcik. Pulmonary hyalinizing granuloma in HIV/AIDS. Can J Infect Dis Med Microbiol 2007;18(3):305307.
\end{abstract}

A 55-year-old man who was recently diagnosed with HIV/AIDS developed multiple bilateral pulmonary nodules after starting highly active antiretroviral therapy. Workup confirmed the diagnosis of pulmonary hyalinizing granuloma. This is the first described case of pulmonary hyalinizing granuloma in HIV/AIDS, and may represent a rare form of immune reconstitution inflammatory syndrome.

Key Words: HIVIAIDS; Immune reconstitution inflammatory syndrome; Multiple pulmonary nodules; Pulmonary hyalinizing granuloma

Dulmonary hyalinizing granuloma $(\mathrm{PHG})$ is a rare benign condition first described in 1977, which usually manifests as multiple bilateral pulmonary nodules of lamellar hyaline collagen deposits (1). It often presents with mild pleuritic chest pain, cough and dyspnea, or can be asymptomatic, detected incidentally on imaging. PHG presents as single or multiple nodules, and radiographically can resemble primary or secondary malignant lesions. Fewer than 100 cases have been reported in the literature (1-3); there is no effective treatment and little is known about its pathogenesis. It has been associated with many autoimmune conditions, as well as previous tuberculous or fungal infections. It may also represent a chronic, exaggerated immune response to either an infectious or autoimmune trigger. The current report presents the first known described case of PHG in a patient with HIV/AIDS.

\section{CASE PRESENTATION}

A 55-year-old man with newly diagnosed HIV/AIDS was seen in July 2000, complaining of three months of fatigue and mild exertional dyspnea. He had been diagnosed with HIV/AIDS following a febrile illness in April 2000. At diagnosis, his CD4 count was less than 100 cells $/ \mathrm{mm}^{3}$ and his viral load was greater than 500,000 copies/mL. He had since finished treatment for both Pneumocystis carinii pneumonia (PCP) and Helicobacter cinaedi bacteremia. Despite this, he had persistent mild fatigue and dyspnea. He denied fever, chills or sweats, and otherwise felt well.

His past medical history included remote hepatitis B, hypertension, dyslipidemia and paroxysmal supraventricular tachycardia. He was an ex-smoker who had quit five months ago. He admitted to the remote use of intravenous drugs, and had both female and male sexual contacts. He had no previous rheumatological or autoimmune conditions and no history of travel or occupational exposures. He was taking trimethoprimsulfamethoxazole and azithromycin for prophylaxis of PCP

\section{Un granulome pulmonaire hyalinisant dans le VIH-sida}

Un homme de 55 ans chez qui on avait récemment diagnostiqué le VIHsida a développé de multiples nodules pulmonaires bilatéraux après avoir entrepris une antivirothérapie hautement active. Le bilan a confirmé le diagnostic de granulome pulmonaire hyalinisant. C'est le premier cas décrit de granulome pulmonaire hyalinisant dans le VIH-sida, et il pourrait constituer une forme rare de reconstitution immune du syndrome inflammatoire.

and disseminated Mycobacterium avium complex infection, respectively.

His physical examination was normal; he was not hypoxic or febrile, and there was no clubbing or pulmonary findings. Pulmonary function tests showed mild obstructive lung disease. Chest radiography revealed a single, asymmetric opacity in the right upper lobe adjacent to the right hilum that was not present in April 2000, at the time of diagnosis of HIV/AIDS. Radiological interpretation suggested that it was either a composite shadow, a focal atelectasis or a focal inflammatory infiltrate. The following week, a repeat chest $\mathrm{x}$-ray showed that the opacity was less prominent, and he received no treatment.

It was thought that his subjective feeling of dyspnea was related to his recent PCP infection. In July 2000, he was started on highly active antiretroviral therapy (HAART), consisting of zidovudine, lamivudine and ritonavir-boosted indinavir.

Due to the patient's ongoing mild shortness of breath and fatigue, a repeat chest x-ray was performed one month later, showing the persistence of the right upper lobe opacity. It appeared spiculated and measured approximately $1.5 \mathrm{~cm}$ in diameter. At this time, the patient's viral load had decreased to 2681 copies/mL and his CD4 count was 396 cells $/ \mathrm{mm}^{3}$.

In October 2000, the patient's radiography (Figure 1) and computed tomography (Figure 2) of the chest revealed multiple, bilateral, smooth, well-defined pulmonary nodules affecting all lobes. The larger of the nodules had a perihilar distribution and there were also multiple subpleural nodules. There were no hilar adenopathy or pleural effusions. Clinically, the patient's respiratory symptoms had improved; his viral load had dropped to 214 copies $/ \mathrm{mL}$ and his CD4 count was 484 cells $/ \mathrm{mm}^{3}$. He declined further investigations.

Repeat chest radiography in February and March 2001 showed marked progression in the size and number of the bilateral pulmonary nodules. The patient denied worsening of his

${ }^{1}$ Internal Medicine Training Program; ${ }^{2}$ Division of Anatomical Pathology; ${ }^{3}$ Division of General Medicine, University of Ottawa, Ottawa Hospital, Ottawa, Ontario

Correspondence and reprints: Dr Theresa Liu, Internal Medicine Training Program, Ottawa Hospital, Box 210, LM - 13, General Campus,

501 Smyth Road, Ottawa, Ontario K1H 8L6. Telephone 613-719-1525, fax 613-737-8250, e-mail TLIU017@uottawa.ca

Received for publication February 27, 2007. Accepted July 18, 2007 


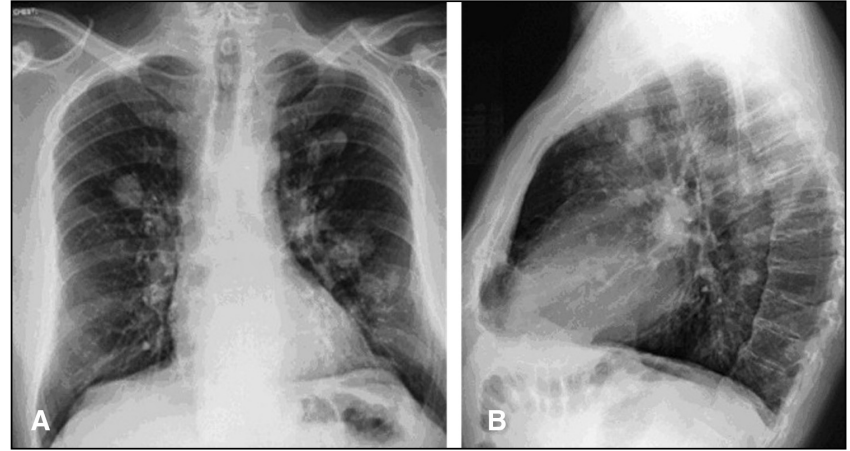

Figure 1) Posteroanterior (A) and lateral (B) chest radiographs of multiple pulmonary nodules

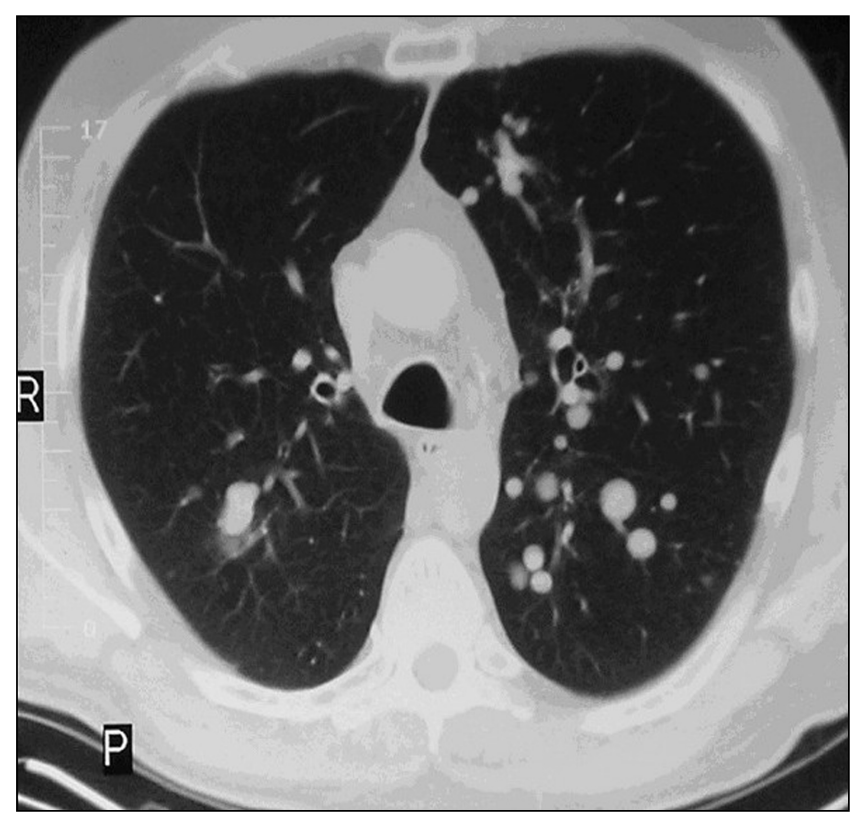

Figure 2) Computed tomography image of multiple bilateral pulmonary nodules. P Posterior; $R$ Right

dyspnea; his viral load was less than 50 copies/mL and his CD4 count was 612 cells $/ \mathrm{mm}^{3}$ on HAART. Bronchial wash and transbronchial biopsy of a left-sided nodule performed in March 2001 were negative for infectious organisms, granuloma and malignancy. In May 2001, a fine needle aspirate biopsy from a lingular nodule showed an aspirate of scant cellularity consisting of red blood cells, benign bronchial cells, pulmonary macrophages and multinucleated histiocytes. A repeat fine needle aspirate biopsy of a right upper lobe nodule in August 2001 was similar to the first, but the material was too scant to perform special stains to rule out infection. In August 2001, a core biopsy of the right upper lobe lesion showed necrotic tissue partially surrounded by chronic inflammatory cells. Special stains were negative for acid-fast bacilli and fungal organisms.

In September 2001, a lingular segment measuring $6 \mathrm{~cm} \times$ $5.7 \mathrm{~cm} \times 2 \mathrm{~cm}$ was excised (Figure 3 ). Microscopic examination revealed multiple hyalinizing granulomas with keloidlike collagen in the centre (Figure 4). Blood vessels and small bronchi were entrapped within the hyalinizing nodules. Chronic inflammatory cells with lymphoid follicles were seen at the nodules' periphery. Special stains for acid-fast bacilli and fungi were negative. Congo red stain was negative for amyloid.

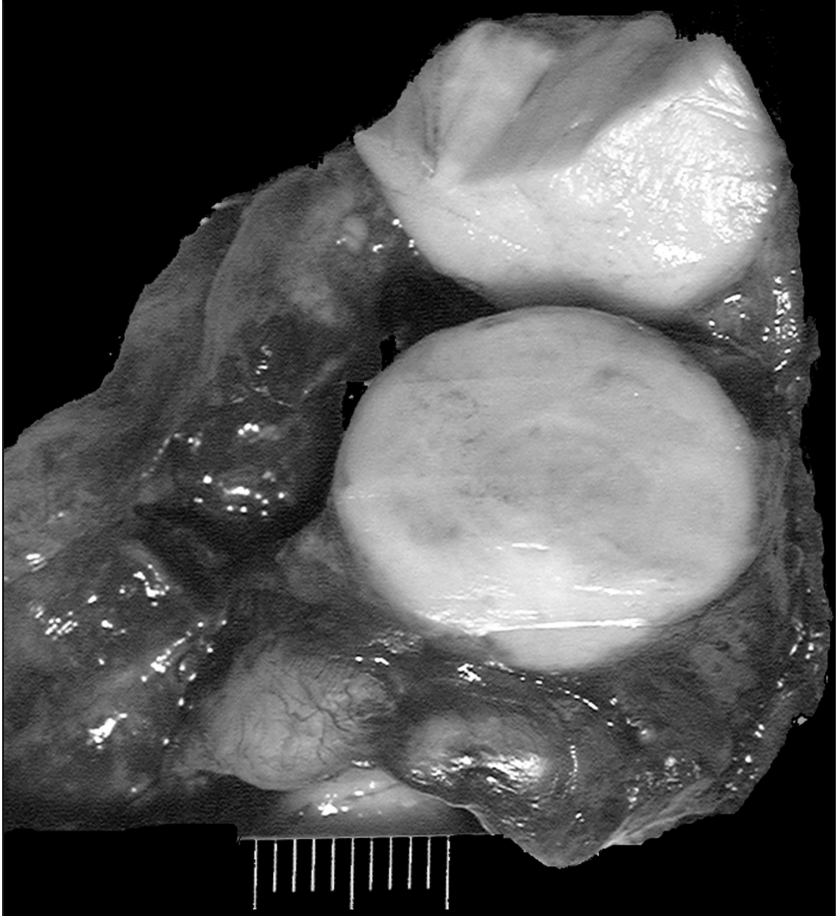

Figure 3) Excised lingular segment with multiple granulomas

Despite the negative fungal stains, on review, the pathologist thought that the nodules could represent a fungal infection, given the patient's profound immunosuppression before starting HAART. However, an empirical three-month course of itraconazole did not improve the patient's mild respiratory symptoms or alter the radiographic appearance of the nodules. It was thought that the original diagnosis of PHG was correct. A trial of corticosteroids was considered but not initiated given the patient's minimal symptoms. Follow-up chest radiography five years later was unchanged.

\section{DISCUSSION}

PHG has no sex, race or age predilection, and usually presents with multiple bilateral pulmonary nodules (1). Patients can be asymptomatic, or complain of mild respiratory symptoms (cough, dyspnea, hemoptysis and pleuritic chest pain) or other nonspecific symptoms (fatigue, fever and weight loss). If present, symptoms are usually nonprogressive. The nodules are slowly progressive in size and number, and there is no specific treatment. Single large nodules are sometimes amenable to surgical resection. Radiographically, the differential diagnosis includes many causes of multiple bilateral pulmonary nodules, including malignancy, granulomatous diseases such as Wegener's granulomatosis or sarcoidosis, amyloidosis, rheumatoid nodules and inflammatory pseudotumour, as well as infectious causes such as tuberculosis (TB) or histoplasmosis $(1,2,4,5)$

Pathology of the lesions in PHG shows concentric, whorled, lamellar hyaline deposits surrounded by plasma cells and lymphocytic infiltrate; early nodules show a predominance of the cellular components and older lesions show more lamellar collagenous bands $(1,2)$. The presence of thick hyalinized collagen bands arranged in whorls, parallel arrays or in a vague storiform pattern is characteristic of PHG. Unlike 
pulmonary sarcoidosis, lesions in PHG show central hyalinization and lack the epithelioid cells seen in the noncaseating epithelioid cell granulomas of sarcoid. Nodular amyloidosis can be excluded by Congo red staining.

Case reports $(2,4,5)$ of PHG have described associations with prior TB, histoplasmosis and Aspergillus pulmonary infections. Others (1-5) have described associations with autoimmune conditions such as rheumatoid arthritis, multiple sclerosis, uveitis, Castleman's disease, sclerosing mediastinitis and retroperitoneal fibrosis. More than one-half of the cases in the literature have positive autoimmune serology (antinuclear antibodies, rheumatoid factor, antismooth muscle antibody, antithyroglobulin antibodies or circulating immune complexes) even in the absence of a primary autoimmune condition $(2,4)$. One case in the literature described symptomatic relief with corticosteroids (5).

The etiology of PHG is unclear. PHG may represent a hyperimmune condition triggered by previous pulmonary infection or a systemic immune response secondary to collagen vascular or autoimmune disease, much as sclerosing mediastinitis and retroperitoneal fibrosis themselves can develop in response to histoplasmosis and TB $(1,5,6)$.

Our patient's multiple bilateral lung nodules were documented on computed tomography three months after beginning HAART; it is unclear whether the patient's isolated solitary right upper lobe nodule on chest $\mathrm{x}$-ray one month before initiating HAART was the same process or a coincidental finding (given the nonspecific pathology of that lesion). His subjective respiratory complaints did improve even as imaging documented progression of his pulmonary nodules over the fall of 2000.

In the present case, it is possible that $\mathrm{PHG}$ could represent a manifestation of noninfectious immune reconstitution inflammatory syndrome (IRIS). IRIS is the pathological inflammatory response to either a previously treated or subclinical infection, temporally associated with the initiation of HAART and subsequent CD4 count recovery and virological suppression (7). IRIS was first described in 2000 by DeSimone et al (7), and typically occurs within weeks to months after the initiation of HAART. It occurs in response to previously treated or subclinical infection with TB, PCP, herpes virus, cytomegalovirus and Mycobacterium avium complex. PHG in the setting of HIV/AIDS may represent IRIS, possibly due to prior exposure to TB or fungal pathogens (which itself may be a risk factor for the development of IRIS).

A recent review (8) suggests that IRIS can occur not only with the reactivation of a latent infection, but also with either a de novo or a previously undetected autoimmune condition such as sarcoidosis, rheumatoid arthritis and Graves' disease (8). Granulomatous diseases, such as sarcoidosis, may serve as a model with which to explain the pathogenesis of PHG in our patient. Sarcoidosis results from an exaggerated cellular immune response (possibly secondary to previous Mycobacterium infection), causing CD4+ type 1 lymphocytes to trigger ongoing and sustained $\mathrm{T}$ lymphocyte triggering, proliferation and activation, in turn causing the attraction and activation of macrophages that induce granuloma formation (9). A deficiency of CD4+ cells (as seen in advanced HIV/AIDS) may inhibit granuloma formation in sarcoidosis because $\mathrm{T}$ lymphocytes are not present in sufficient quantities to accumulate in affected tissues. Similarly, the development of PHG in a patient beginning HAART could be a manifestation

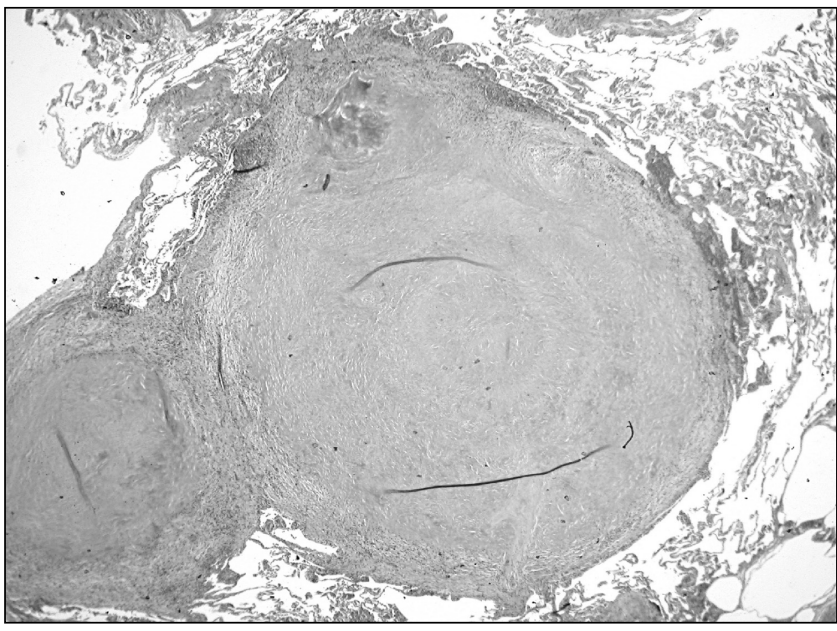

Figure 4) Low magnification view of well-circumscribed pulmonary hyalinizing granuloma with central keloid-like collagen

of his recovering CD4+ count and partial restoration of immune function.

\section{CONCLUSION}

PHG is a rare, benign, immune-mediated lung disease with no specific treatment. To our knowledge, this is the first described case of PHG in a patient with HIV/AIDS. Because the diagnosis of PHG was temporally preceded by the initiation of HAART, it is possible that it could have been due to an immune reconstitution phenomenon. It is well known that patients with HIV/AIDS are at risk for usual common pulmonary infections, as well as TB, PCP and other fungal pneumonias that can present as multiple pulmonary nodules. However, PHG should also be considered in the differential diagnosis of multiple pulmonary nodules in the setting of treated HIV/AIDS.

\section{REFERENCES}

1. Engleman P, Liebow AA, Gmelich J, Friedman PJ. Pulmonary hyalinizing granuloma. Am Rev Respir Dis 1977;115:997-1008.

2. Ren Y, Raitz EN, Lee KR, Pingleton SK, Tawfik O. Pulmonary small lymphocytic lymphoma (mucosa-associated lymphoid tissue type) associated with pulmonary hyalinizing granuloma. Chest 2001;120:1027-30.

3. Lvovsky D, Rosman J, Iliescu ME. Pulmonary hyalinizing granuloma (PHG) presenting as bilateral dense apical masses. Chest 2004;126:950S.

4. Esme H, Ermis SS, Fidan F, Unlu M, Dilek FH. A case of pulmonary hyalinizing granuloma associated with posterior uveitis. Tohoku J Exp Med 2004;204:93-7.

5. Shinohara T, Kaneko T, Miyazawa N, et al. Pulmonary hyalinizing granuloma with laryngeal and subcutaneous involvement: Report of a case successfully treated with glucocorticoids. Intern Med 2004:43:69-73

6. Wheen LC, Taylor GB. Pulmonary hyalinizing granuloma: An airway-acquired etiology? Pathology 2004;36:583-5.

7. DeSimone JA, Pomerantz RJ, Babinchak TJ. Inflammatory reactions in HIV-1-infected persons after initiation of highly active antiretroviral therapy. Ann Intern Med 2000;133:447-54.

8. Calabrese LH, Kirchner E, Shrestha R. Rheumatic complications of human immunodeficiency virus infection in the era of highly active antiretroviral therapy: Emergence of a new syndrome of immune reconstitution and changing patterns of disease. Semin Arthritis Rheum 2005;35:166-74.

9. Trevenzoli M, Cattelan AM, Marino F, Marchioro U, Cadrobbi P. Sarcoidosis and HIV infection: A case report and review of the literature. Postgrad Med J 2003;79:535-8. 


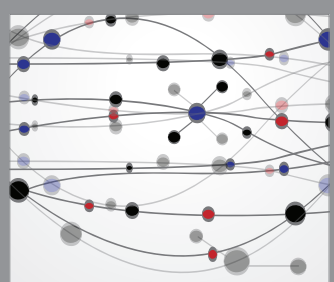

The Scientific World Journal
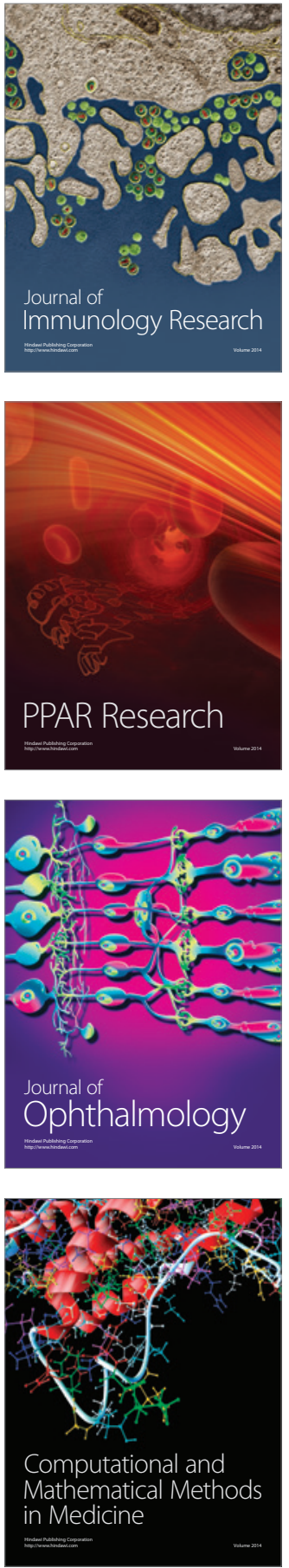

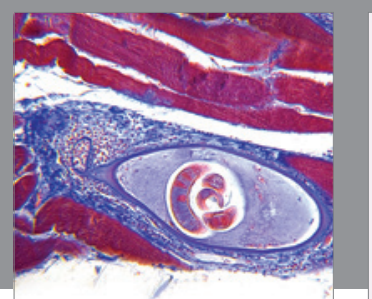

Gastroenterology Research and Practice

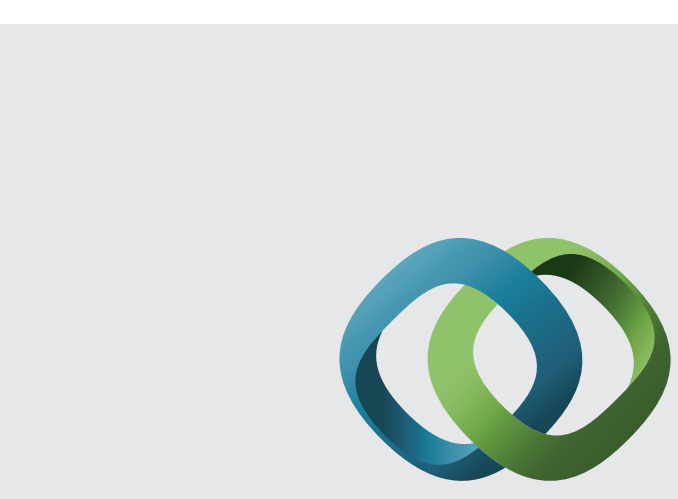

\section{Hindawi}

Submit your manuscripts at

http://www.hindawi.com
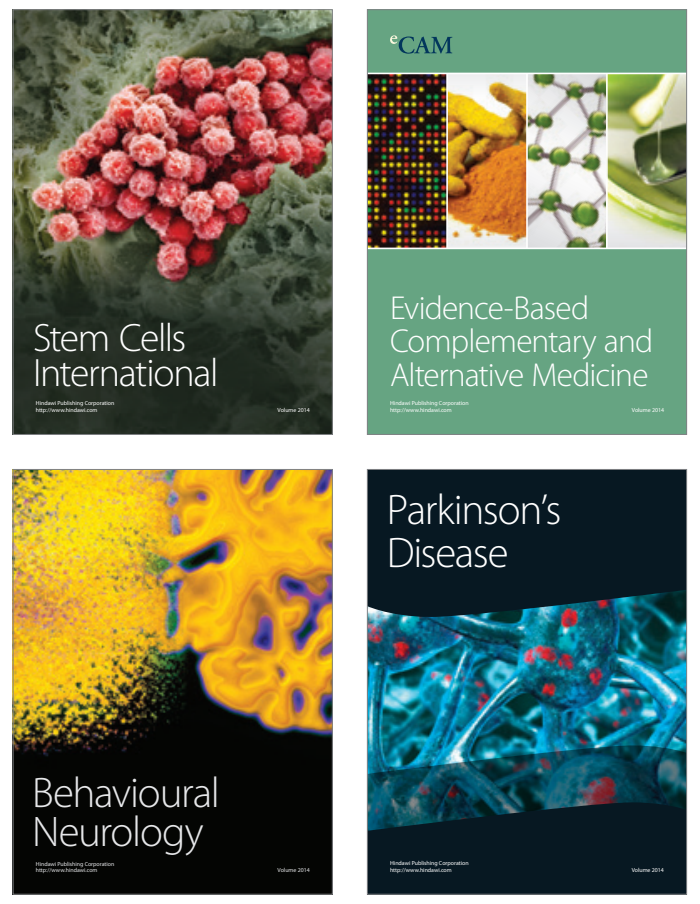
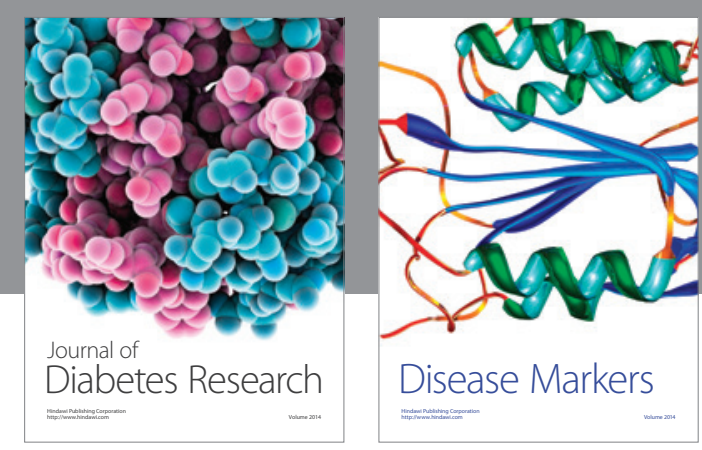

Disease Markers
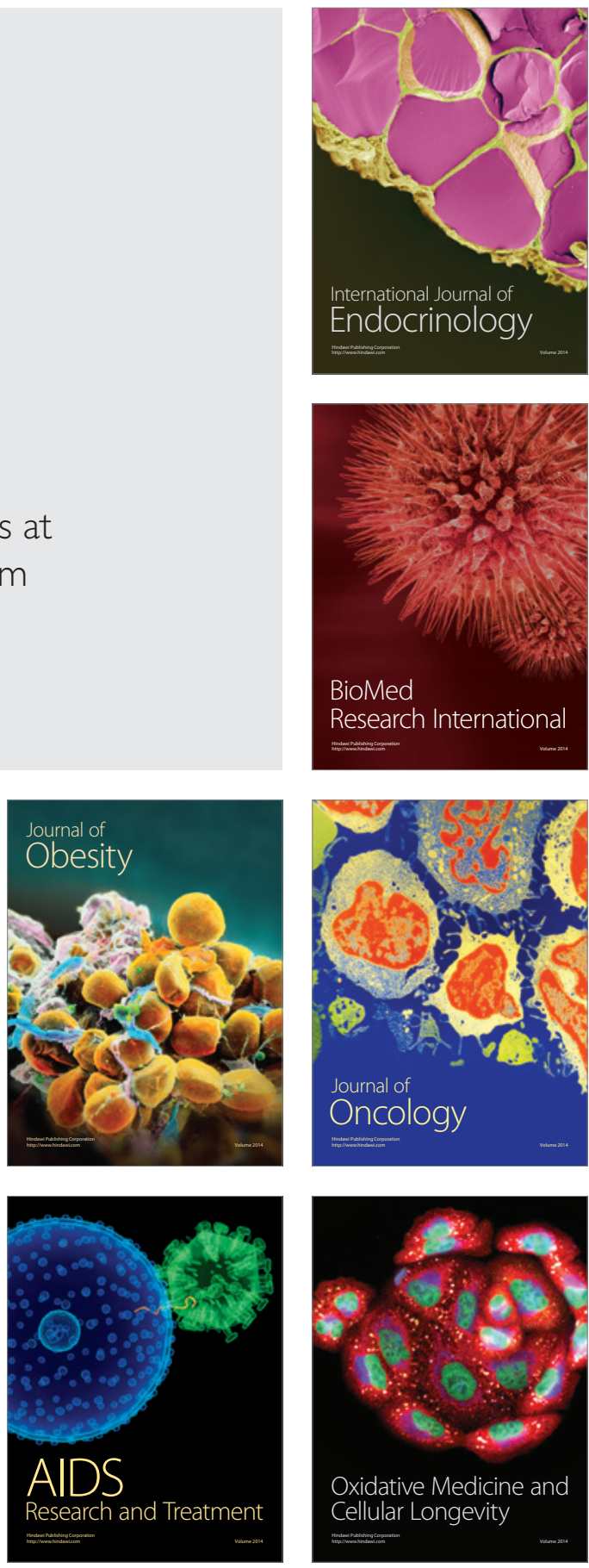